\title{
Numerical simulation of flake orientation during droplet impact on substrates in spray painting processes
}

\author{
Q. Ye ${ }^{\star 1}$, M. Dreher ${ }^{1}$, O. Tiedje ${ }^{1}$, B. Shen ${ }^{2}$, J. Domnick ${ }^{2}$ \\ ${ }^{1}$ Fraunhofer Institute for Manufacturing Engineering and Automation, Stuttgart, Germany \\ ${ }^{2}$ University of Applied Sciences Esslingen, Esslingen, Germany \\ *Corresponding author email: Qiaoyan.ye@ipa.fraunhofer.de
}

\begin{abstract}
A numerical study of flake orientation by droplet impact on dry and wetted solid surfaces for spray painting processes has been carried out. A dynamic contact angle model was applied for the calculation of viscous droplet impact on the dry surface after experimental validation. A user-defined 6-DOF model that concerns to the rigid body motion was implemented in a commercial CFD program to calculate the flake movement inside the droplet. The simulated flake orientations show interesting results that are helpful to understand and to improve painting processes.
\end{abstract}

\section{Keywords}

Droplet impact, rigid body dynamics, flake orientation.

\section{Introduction}

Effect pigments (flat, $5-40 \mu \mathrm{m}$ broad flakes) are widely used in industrial spray painting processes for imparting colour and coating appearance, enabling e.g. metallic effect coatings that are very popular in the automotive industry. From practical observations, it is already well known that the initial pigment/flake orientation in the paint layer, primarily at the early stage of the film formation and before the subsequent solvent evaporation and baking, influences the final metallic effect significantly. Therefore, it is strongly suspected that processes during viscous droplet impact have a decisive influence on pigment orientation. For a strong effect, usually a pigment orientation parallel to the substrate is desired.

Regarding the droplet impact dynamics, there have been already many research works [1-8]. In experimental studies, mainly large droplets (e.g. D > 500 $\mu \mathrm{m}$ ) with low viscosity under hydrophilic/hydrophobic surface conditions were investigated. Different outcomes of droplet impact on dry/wet substrates were analysed [4]. The air entrapment by droplet impact was studied [3]. The maximal droplet-spreading diameters were correlated with non-dimensional numbers, i.e. Weber and Reynolds numbers, using published experimental results [5]. The contact angle models that have to be applied in the numerical simulation were studied and discussed in detail $[7,8]$.

There are not so many studies that focus on the flake movement inside the droplet and in the film. For small droplets $(50-300 \mu \mathrm{m})$, especially for opaque liquids, like in spray painting processes, it is very difficult to obtain high quality time-resolved imaging of the flake orientation during droplet impingement experimentally. Although a few predictions of flake orientation were carried out based on some mathematical analysis [9] and the flow field of twodimensional droplet impact calculation [10], the corresponding results should be further verified. The objective of the present paper is to carry out a detailed numerical study of flake orientation, focusing on droplet impact processes on dry and wetted solid walls. Thereby, a contact angle model that is necessary in the numerical simulation was developed for the paint droplet impact process. In addition, a user-defined 6DOF (6-degrees-of-freedom) solver was 
implemented in a CFD-program to perform the rigid body (flake) motion calculation within the impacting droplet. The developed models were applied in a parameter study, to further clarify the existing dependencies on application and fluid parameters more quantitatively.

\section{Basic numerical methods}

The droplet impact and spreading on a surface is an example of an interfacial flow problem that can be calculated using the Volume of Fluid (VOF) method. Hereby, two or more immiscible fluids can be modelled by solving a single set of momentum and mass equations and tracking the volume fraction of each of the fluids throughout the domain. The numerical simulations in this work were carried out with the commercial CFD code ANSYS-FLUENT based on the finite-volume approach.

Time dependent VOF calculations were performed using an explicit scheme. A geometric reconstruction scheme for the volume fraction discretization was used, ensuring a sharp and low-diffusion interface discretization. To accommodate surface tension effects, the CSF-model with wall adhesion modelling was used. The PRESTO scheme was applied for the pressure discretization. For pressure-velocity coupling, the coupled algorithm had to be applied, since there exists a compatibility constraint whilst using the overset mesh model for flake movement calculation. Cartesian grids with local mesh-refinement in the relevant regions were created. It is well known that the VOF-method is quite sensitive to grid resolution. Therefore, a study of grid independence was performed by comparing measured and calculated spread and height factors of the droplet impact. A reasonable cell size was obtained at about $D / \Delta x=150$ and applied around the droplet and liquid film on the substrate. Here $D$ denotes the droplet diameter and $\Delta x$ the grid size. In the far-field, coarser hexahedral meshes were used to reduce the total number of cells and therefore computational cost. Consequently, the domains in the present study contain 20 to 150 million cells.

For the present study, a single droplet containing a single rectangular shaped flake is applied. The corresponding important boundary conditions and models are described as follows.

\section{Simulation of viscous droplet impact on dry solid surfaces}

A computational domain of $2 \mathrm{D} \times 2 \mathrm{D} \times 1.5 \mathrm{D}$ was created for the simulation of droplet impact on the dry solid surface. Thereby, only a quarter of the spherical droplet was calculated and symmetry boundary conditions were used to reduce the total amount of cells. A flow field aware variable time step with the CFL (Courant-Friedrichs-Lewy) number $<1$ was set. The resulting time step sizes ranged from $5 \mathrm{e}-9$ to $1 \mathrm{e}-7 \mathrm{~s}$. Based on common spray-painting conditions, droplet impact velocities between $0.5-12 \mathrm{~m} / \mathrm{s}$ were applied. The initial droplet position was chosen to be a few micrometers above the wall surface, so that the surrounding gas field can be calculated, which is absolutely necessary in the study of droplet impact dynamics. The pressure inside the droplet, induced by the surface tension of the liquid, was calculated and properly initialized. The remaining domain is set to ambient pressure. A dry smooth wall with no-slip boundary condition was used.

It is well known that the description of moving contact line or contact angle in the numerical simulation of droplet impact on dry surfaces is not trivial and quite challenging. Nevertheless, dynamic contact angles are widely used in VOF-simulations to accommodate adhesion behaviour. However, the applicability of the corresponding models is quite problem dependent, such as the variety of liquid and substrate properties, as well as the numerous 
operating parameters. The hysteresis of contact line and contact angle makes the model development more difficult. Further information can be referred to $[7,8]$.

We have carried out the experimental observation of viscous droplet impact on dry solid surfaces, focusing on the contour evolution during the spreading and receding processes. The experimental results provided useful information to develop the model for dynamic contact angle that was applied as boundary condition in the simulation. Equation 1 describes the model in a mathematical manner.

$$
\theta_{D}=\left\{\begin{array}{cc}
v_{c l}>0 & \theta_{A} \\
\overline{v_{c l}}<|\delta| \cap v_{c l}<\delta & \theta_{\text {int }}=90^{\circ} \\
\theta_{\nabla \phi}<\theta_{A} & \theta_{\nabla \phi} \\
\theta_{\nabla \phi} \leq \theta_{E} & \theta_{E}
\end{array} \quad \text { where } \delta=-0.1\right.
$$

In the spreading phase, where the contact line velocity $\left(v_{c l}\right)$ is significantly positive, a constant advancing contact angle $\left(\theta_{A}\right)$ is deployed. At maximum wetting spread, when the contact line movement stops and the droplet usually starts to recede, an intermediate contact angle $\left(\theta_{\text {int }}\right)$ is set. This helps to suppress contact line movement in accordance to the experimental observation. If the angle between the fluid interface and the wall, calculated by the gradient of the VOF-field $(\nabla \phi)$, gets smaller than the advancing contact angle, the interphase-angle is set as contact angle $\left(\theta_{\nabla \phi}\right)$. It is secured, that this angle decreases monotonically to the static or equilibrium contact angle $\left(\theta_{E}\right)$. On the wall cells and far away from the contact line, the equilibrium contact angle $\left(\theta_{E}\right)$ is used, which ensures the wetting behaviour on the wall is more reasonable.

\section{Simulation of viscous droplet impact on wet solid surfaces}

A thin film with height $H_{f}=60 \mu \mathrm{m}$ and infinitive width was applied in the present simulation. Thereby, a computational domain of $8 \mathrm{D} \times 8 \mathrm{D} \times 3 \mathrm{D}$ was created for the simulation of droplet impact on the wet solid surface. Clearly, for large droplet, the number of computational cells will be increased tremendously. In this paper, we show only the simulation by using droplet with the diameter of $50 \mu \mathrm{m}$. Local grid adaption was carefully performed, ensuring the necessary grid resolution.

\section{Simulation of flake orientation}

For the simulation of flake movement, a dynamic mesh model in conjunction with the overset meshing method of ANSYS Fluent was applied. Hereby, a component mesh for the flake and the background mesh, representing the domain, are overlaid and connected via an interpolated mesh interface. In comparison to other methods, the resulting elimination of distorted cells and consequently the necessity of remeshing yields VOF-friendly meshing setup and a good performance in the flake movement calculation. The size of the considered flake was chosen to be $1 \times 16 \times 16 \mu \mathrm{m}^{3}$ that is a reasonable estimate concerning typical effect pigment size distributions. Yet, this results in corresponding moments of inertia of $1.6 \times 10^{-23}$ $\left[\mathrm{kg} \cdot \mathrm{m}^{2}\right]$, which are too marginal for the currently available 6DOF motion solver by ANSYS. In consequence, a custom motion equation solver has been implemented via a user-definedfunction (UDF). This 6DOF solver has been developed under the assumption of rigid body motion. Therefore, the flake motion may be simplified to the movement of its centre of mass and the rotation around it. Consequently, the momentum conservation (2) is calculated in the 
global inertial coordinate system, and the angular momentum (3) is determined in body coordinates:

$$
\begin{aligned}
& \dot{\boldsymbol{v}}=\frac{1}{m} \sum \boldsymbol{F} \\
& \dot{\boldsymbol{\omega}}=\boldsymbol{I}^{-1}\left(\sum \boldsymbol{M}-\boldsymbol{\omega} \times \boldsymbol{I} \boldsymbol{\omega}\right)
\end{aligned}
$$

Here, $m$ denotes the mass, $\boldsymbol{I}$ the inertia tensor and $\boldsymbol{F}, \boldsymbol{M}$ the flow induced forces and moments, respectively. The points over the velocity $(\boldsymbol{v})$ and the angular velocity $(\boldsymbol{\omega})$ represent time derivatives. In equation (2), the sum includes pressure, viscosity and gravitational forces. An Adams-Moulton algorithm of $4^{\text {th }}$ order with a rather complex variable time step formulation has been derived and implemented to time integrate the above given ODEs. This enables simulations of flake movement or orientation stable with a quite reasonable time step size, such as $\mathrm{dt}=1 \mathrm{e}-8$ to $1 \mathrm{e}-7 \mathrm{~s}$ that is solely adjusted by the flow solver.

\section{Results and Discussion}

Considering the shear thinning and thixotropic non-Newtonian behaviour of most paint liquids, fluids with a constant viscosity between 20 - $40 \mathrm{mPas}$ were chosen that represent a good approximation of the high shear viscosity of typical paint materials. Moreover, a surface tension of $0.025 \mathrm{~N} / \mathrm{m}$ was used in the simulations. Depending on different atomizer and droplet size, the droplet impact velocity could be from 0.5 to $50 \mathrm{~m} / \mathrm{s}$ [11]. Nevertheless, velocity $<12$ $\mathrm{m} / \mathrm{s}$ was used, since a representative mean diameter of paint droplet is usually smaller than $50 \mu \mathrm{m}$. Table 1 summarizes some parameters used in this study.

Table 1. Parameters used in the simulation for Newtonian drops

\begin{tabular}{|l|l|l|l|}
\hline Droplet diameter $\mathrm{D}(\mu \mathrm{m})$ & 50,300 & Height of film $(\mu \mathrm{m})$ & 60 \\
\hline Droplet velocity $\mathrm{U}(\mathrm{m} / \mathrm{s})$ & $0.5-12$ & Flake size $\left(\mu \mathrm{m}^{3}\right)$ & $1 \times 16 \times 16$ \\
\hline Liquid viscosity $\mu(\mathrm{Pas})$ & $0.017,0.02,0.04$ & Flake density $\left(\mathrm{kg} / \mathrm{m}^{3}\right)$ & 3200 \\
\hline Surface tension $\sigma(\mathrm{N} / \mathrm{m})$ & $0.025,0.063$ & Ratio of flake to droplet l/D & $0.053,0.32$ \\
\hline Liquid density $\rho\left(\mathrm{kg} / \mathrm{m}^{3}\right)$ & 1020,1200 & Re-number, $\mathrm{Re}=\rho \mathrm{UD} / \mu$ & $5-100$ \\
\hline Static contact angle $\left(^{\circ}\right)$ & $50-60$ & We-number, $\mathrm{We}=\rho \mathrm{U}^{2} \mathrm{D} / \sigma$ & $3-1200$ \\
\hline
\end{tabular}

Validation of simulation of droplet impact on dry solid surface

At first, validations of the numerical models were carried out for the impact process of viscous droplet on dry solid surfaces. Thereby, using a droplet generator, glycerol/water droplets (D = $\left.400 \mu \mathrm{m}, \mu=20 \mathrm{mPas}, \sigma=0.063 \mathrm{~N} / \mathrm{m}, \theta_{\mathrm{E}}=55^{\circ}\right)$ and paint droplets $(\mathrm{D}=300 \mu \mathrm{m}, \mu=17 \mathrm{mPas}$, $\sigma=0.025 \mathrm{~N} / \mathrm{m}, \theta_{\mathrm{E}}=53^{\circ}$ ) were created. Subsequently, the droplet spread-factors $d / D_{0}$ and height ratios $h / D_{0}$ induced by the impact process were recorded and measured using a highspeed camera. The advancing angles in the simulation were set for glycerol/water droplet $\theta_{\mathrm{A}}$ $=120^{\circ}$ and paint droplet $\theta_{A}=95^{\circ}$, respectively. Figure 1 shows a comparison of these values between the experimental data and the simulation results. A quite good agreement can be observed, which indicates that the proposed dynamic contact angle model delivers good performance and is suitable for the present application.

\section{Flake orientation by droplet impact on dry solid surfaces}

The position and orientation of effect pigments within a droplet may be randomly distributed. For the present simulation, the flake has initially been located at the vertical centre at one half of the droplet radius and oriented vertically to the solid surface. This initial orientation angle is considered as worst-case whilst a horizontal orientation as optimal in the resulting film. In order words, the ideal flake is aligned parallel to the solid surface after the curing process. 

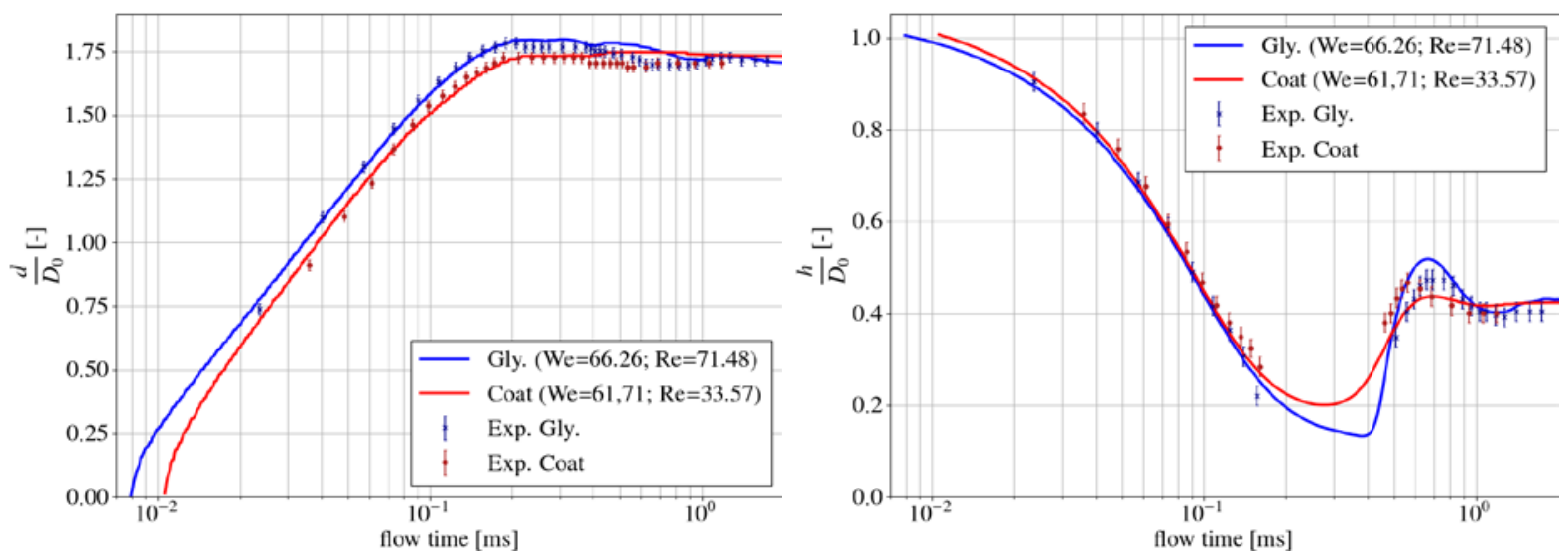

Figure 1. Comparison of droplet spread-factors between experiment and simulation during the impact process.

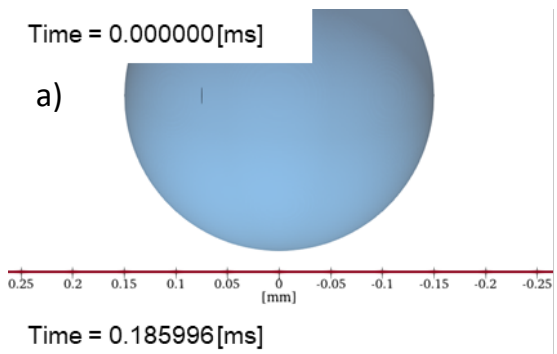

Time $=0.099958[\mathrm{~ms}]$

b)

c)
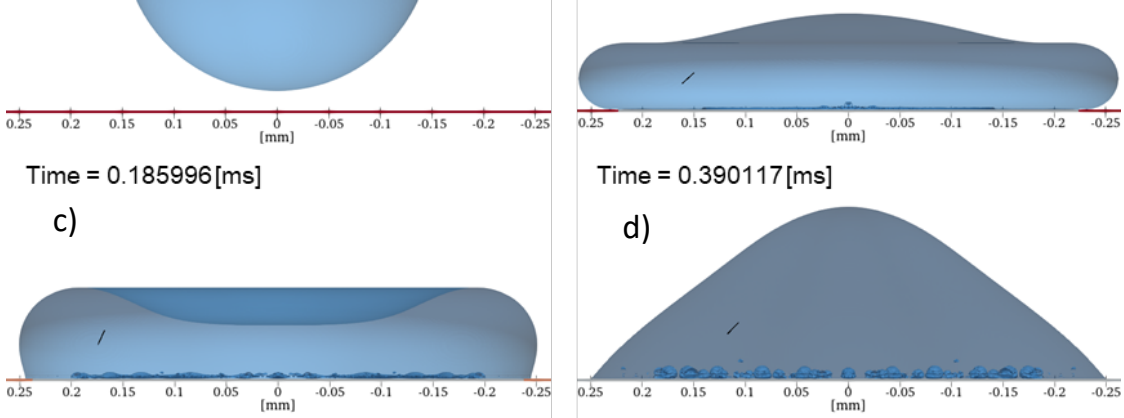

Time $=0.390117[\mathrm{~ms}]$

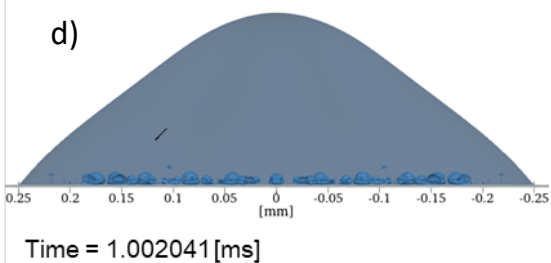

Time $=0.702077[\mathrm{~ms}]$
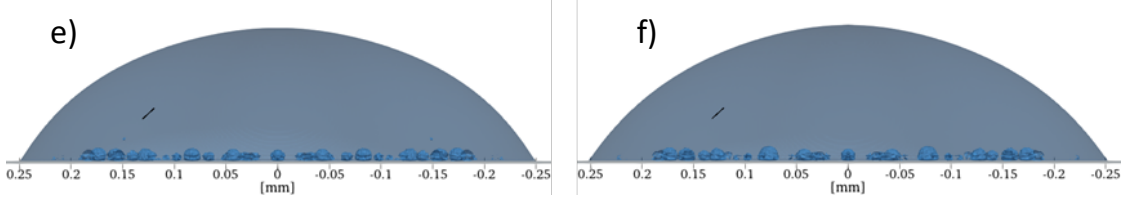

Figure 2. Evolution of the droplet contour and flake orientation $(D=300 \mu \mathrm{m}, \mu=20 \mathrm{mPas}, \sigma=0.063 \mathrm{~N} / \mathrm{m}, \mathrm{U}=3$ $\mathrm{m} / \mathrm{s}, \theta_{\mathrm{E}}=55^{\circ}, \mathrm{We}=50, \mathrm{Re}=54$ )

Figure 2 shows the contour evolution and the flake orientation during a droplet impact process. Fig. 2 a) depicts the initial state. The inertia driven spreading phase, as shown in Fig. 2 b), turns the flake quite substantially. In Fig.2 c) the maximum spreading is reached and surface tension effects become dominant. During this stage, despite droplet contour movement, the mentioned contact line hysteresis is observed, which is identical to our experimental findings. Here, the flake is found to increase its orientation angle again. This is partly reversed during the recoiling and whilst the final equilibrium state is reached, as shown in Fig.2 d), e), f). Air entrapment by droplet impact and the air bubble (blue blobs) developing on the wall can be also observed in Fig.2, which is another topic and will not be discussed in the present paper.

The effects of the droplet liquid properties and impact parameters on the flake orientation are obtained and shown in Fig.3. Glycerol/water and paint drops with $\mu=20 \mathrm{mPas}$ were applied. For small droplets, such as $D=50 \mu \mathrm{m}$, the quasi-static state is reached at $t=0.3 \mathrm{~ms}$. Since the size ratio between flake and droplet diameter is relative large, $1 / D=0.32$ for $50 \mu \mathrm{m}$ droplet, the movement of the flake is quite limited. For $300 \mu \mathrm{m}$ droplets, a larger change in orientation angle can be observed. It takes a relative long time to reach the static state for large We- or Re-number. The final flake angle in the quasi-static state depends, presumably, mainly on the 
Re-number. It seems that the flake orientation improves with increasing Re-number, which should be further studied by increasing the parameter variation.

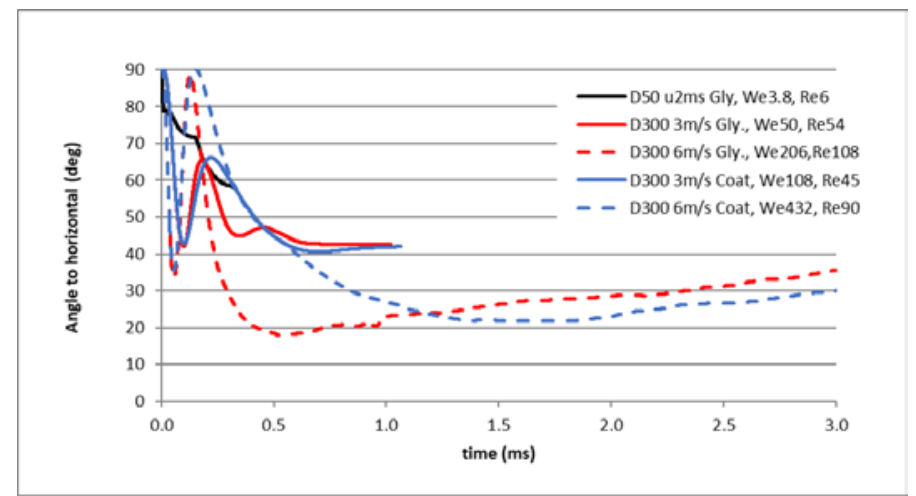

Figure 3. Comparison of flake orientation with different viscous droplets.

The interaction between flake and flow field may be observed more detailed in Figure 4. At $\mathrm{t}=0.17 \mathrm{~ms}$ (Fig. $4 \mathrm{c}$ ), there is no macroscopic contour movement left, which is referred to as quasi-static state. Although the velocity magnitude is quite small, there are eddies arising around the pigment (Fig.4. d, c), resulting in some disorientation of the flake. This effect requires further investigation. Also, the entrapment of air bubbles on the solid surface, which are later noticed also in the paint film, can be seen in Fig. 2 \& 4. But this phenomenon will not be discussed in this paper.
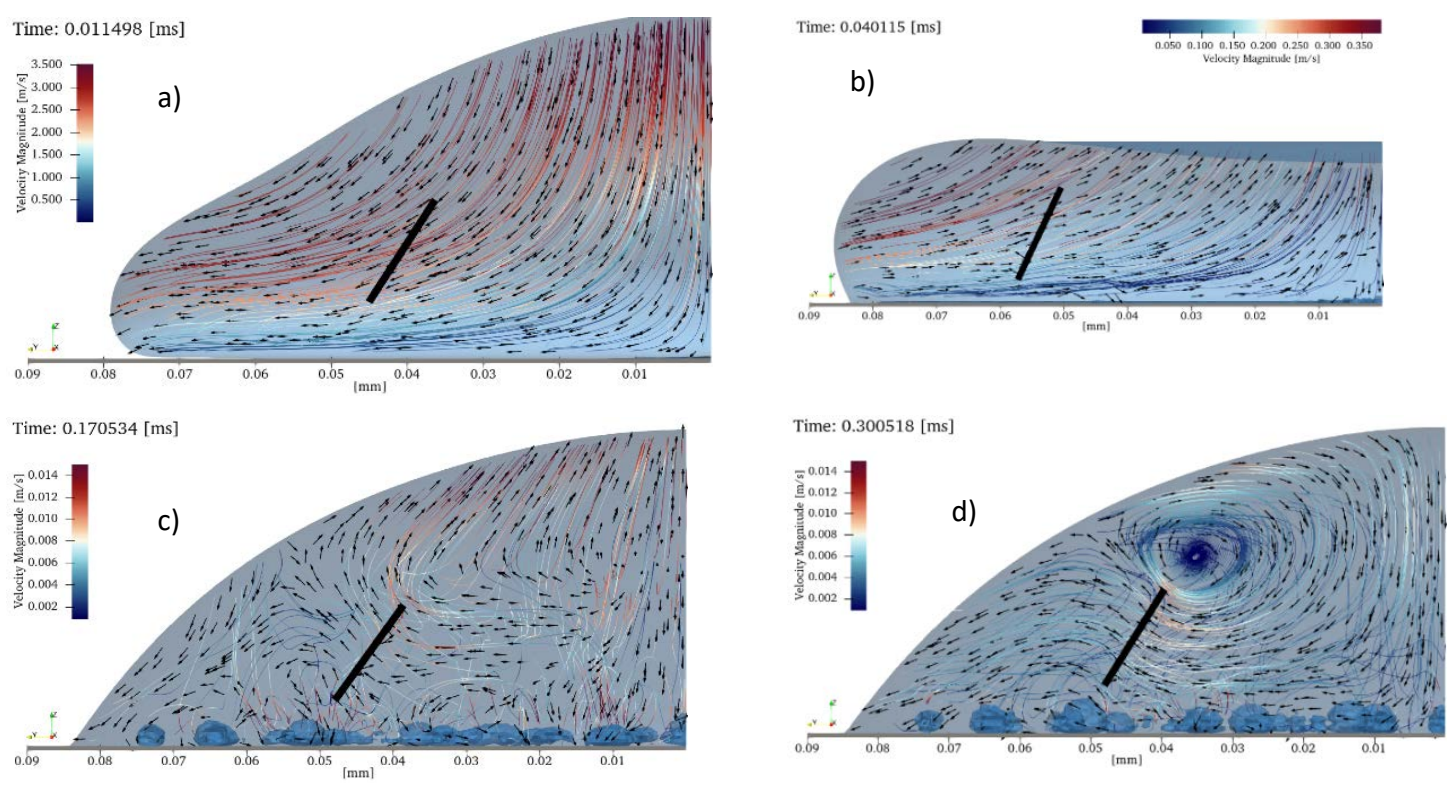

Figure 4. Flake within coat droplet $\left(D=100 \mu \mathrm{m}, v=6 \mathrm{~m} / \mathrm{s}, \mathrm{We}=153.3, \operatorname{Re}=30.7, \theta_{\mathrm{E}}=53^{\circ}\right)$; overlaid with velocity vector $(\mathrm{m} / \mathrm{s})$ and streamline: a) advancing process $\mathrm{b}$ ) receding process, $\mathrm{c}$ ) and d) quasi-static state.

\section{Flake orientation by droplet impact on wet solid surfaces}

Flake orientation by droplet impact on the wet surface is more concerned than on the dry surface, since the optical properties is mainly influenced by flakes located close to the film surface. Depending on the impact velocity and liquid viscosity, basically, the outcome of droplet impact on a thin liquid film can be divided into spreading/deposition, cratering/receding, crown formation and crown with splashing. The latter will not be studied in the present investigation because it is very seldom in spray coating applications. Typical spreading and cratering processes are shown in Fig.5 and Fig. 6, respectively. The crater occurs by 
increasing the impact velocity. With deepening the crater, as shown in Fig.6, the flake angle decrease and even tends to parallel to the solid surface. However, by crater receding the flake angle increases again. Some comparison of flake orientations using different viscous droplets is depicted in Fig.7. At quasi-static state, we can observe that for droplet with lower viscosity (20 mPas) the flake angle with lower impact velocity is smaller $\left(32^{\circ}\right)$ than that $\left(50^{\circ}\right)$ with high impact velocity. The paint droplet $(\mu=40 \mathrm{mPas})$ that undergoes only the spreading process shows the largest flake angle $\left(58^{\circ}\right)$ in Fig.7. The simulations show quite interesting results. Further parameter study, especially with different viscosity and initial flake angle should be carried out.
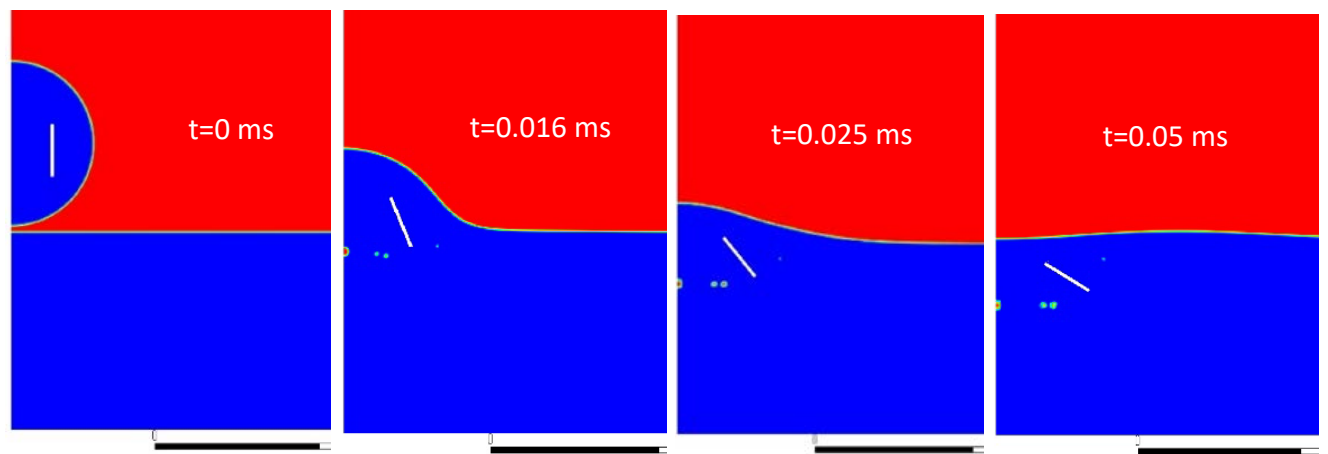

Figure 5. Evolution of droplet-, film-contour, and flake orientation (black ruler corresponds to $50 \mu \mathrm{m}$ ), $\mathrm{D}=50 \mu \mathrm{m}, \mu=$ $20 \mathrm{mPas}, \mathrm{U}=2 \mathrm{~m} / \mathrm{s}, \sigma=0.063 \mathrm{~N} / \mathrm{m}, \mathrm{We}=3.8, \mathrm{Re}=6$.
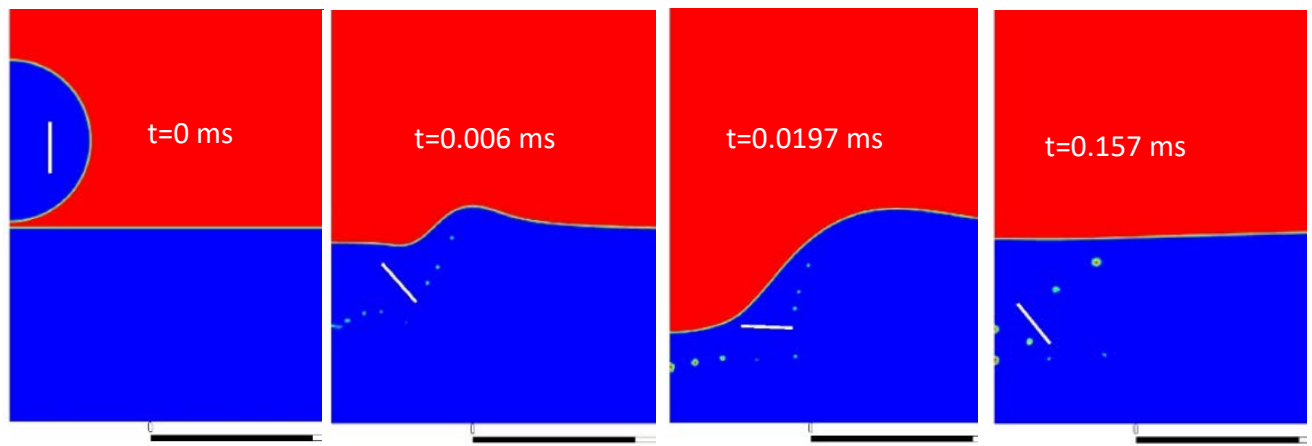

Figure 6. Evolution of droplet-, film-contour, and flake orientation, $D=50 \mu \mathrm{m}, \mu=20 \mathrm{mPas}, \mathbf{U}=10 \mathrm{~m} / \mathrm{s}, \sigma=0.063$ $\mathrm{N} / \mathrm{m}, \mathrm{We}=95, \mathrm{Re}=30$.

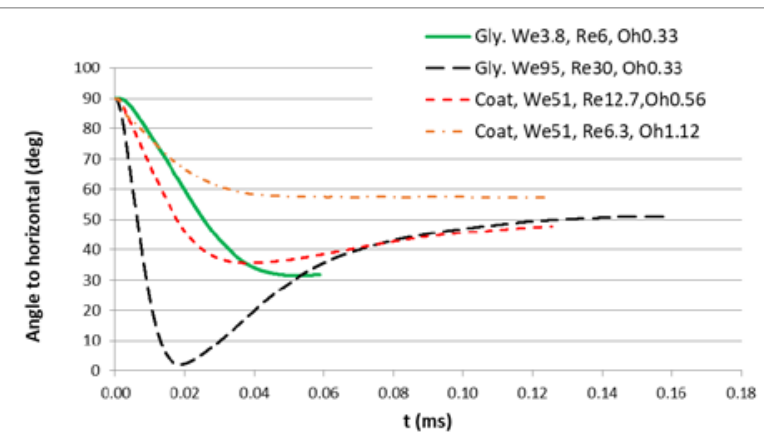

\section{Conclusions}

It is the first time that a detailed numerical study of the flake orientation by the viscous droplet impact on dry/wet solid surfaces has been carried out. A dynamic contact angle model that is suitable to the spray painting process was proposed and validated by the experimental
Figure 7. Comparison of flake orientations by droplet impact on the wet solid surface using different viscous droplets, We, Re and Oh are dimensionless numbers, $\mathrm{Re}$ and We are defined in Table 1, Oh $=\mu / \sqrt{\sigma D \rho}$. 
observations. We have succeeded to implement a rigid body motion solver for calculating pigment movement in ANSYS-Fluent. Some numerical treatments have been applied, ensuring accurate calculation of derivatives and stable solution by solving the flake motion equations, which makes the simulation of the flake movement possible in a practical sense.

A first parameter study was performed. In the case of droplet impact on a dry surface, a relatively large flake angle with respect to the horizontal surface could be still expected after the receding process at quasi-static state, although the flake angle changes tremendously with increasing Re-number. In the case of a droplet impact on a wet surface, for a given low viscous droplet and at quasi-static state, it seems that the flake angle increases with increasing velocity because of the effect of crater receding. It is clearly observed that higher viscosities of droplet and film reduce the flake movement, resulting in smaller differences between the initial and the final flake angles. Further detailed parameter study should be performed, especially with different initial flake angles, finally resulting in stable and reproducible final flake angles as a function of dimensionless numbers.

\section{Acknowledgments}

The present investigations were supported by the German Federal Economical Affairs and Energy through the Arbeitsgemeinschaft industrieller Forschungsvereinigungen (AIF). The simulations were performed in the High Performance Calculation Centre Stuttgart (German federal project: Droplmp). These supports are gratefully acknowledged by the authors.

\section{References}

[1] Chandra, S. and Avedisian, C. T. "On the collision of a droplet with a solid surface", Proc. R. Soc. London, Ser. A 432, 13 (1991).

[2] Thoroddsen, S. T. and Sakakibara, J. "Evolution of the fingering pattern of an impacting drop", Phys. Fluids 10(6):1359-1374 (1998).

[3] Thoroddsen,S. T., Takehara, K. and Etoh, T. G., "Bubble entrapment through topological change", Phys. Fluids 22(051701):1-4 (2010).

[4] Weiss Daniel A., Yarin Alexander L. Single drop impact onto liquid films: neck distortion, jetting, tiny bubble entrainment, and crown formation. Journal of Fluid Mechanics, vol.385: 229-254, 1999

[5] Arogeti, M., Sher, E. and Bar-Kohany, T., A single spherical drop impact on a flat, dry surface - a unified correlation, Atomization and Sprays, 27(9):759-770 (2017).

[6] Kim, E. and Baek, J., Numerical study of the parameters governing the impact dynamics of yield-stress fluid droplets on a solid surface, Journal of Non-Newtonian Fluid Mechnics 173174 (2012) 62-71

[7] Šikalo, Š., Wilhelm, H.-D., Roisman, I. V., Jakirlić, S. and Tropea, C., Dynamic contact angle of spreading droplets: Experiments and simulations, Physics of Fluids 17, 062103 (2005); https://doi.org/10.1063/1.1928828

[8] Linder, N., Criscione, A., Roisman, Ilia V., Marschall, H., Tropea, C., 3D computation of an incipient motion of a sessile drop on a rigid surface with contact angle hysteresis, Theor. Comput. Fluid Dyn. 2015, DOI 10.1007/s00162-015-0362-9

[9] Kirchner, E., Flow-induced orientation of flakes in metallic coatings - II. The orientation mechanism, Progress in Organic Coatings, 124 (2018) 104-109.

[10] Schlüsener, T., Untersuchungen zum Einfluss der thermo- und hydrodynamischen Vorgänge bei der Lackapplikation und -trocknung auf die Farbtonausbildung wasserbasierter Metallic-Lacke, PhD thesis in German, 2000

[11] Ye, Q and Domnick, J., Analysis of droplet impingement of different atomizers used in spray coating processes, J. Coat. Technol. Res., 14 (2) 467-476, 2017. 